\title{
Development of a Multiplex PCR for Discrimination of the TLC:RS1:CTX array of Vibrio cholerae Wave 3 El Tor Strains
}

\author{
Eun Jin Kim ${ }^{1,2 \dagger}$, Hyun Jin $\mathrm{Yu}^{1,2 \dagger}$, G. Balakrish Nair ${ }^{3}$, and Dong Wook Kim ${ }^{1,2 *}$ \\ ${ }^{1}$ Department of Pharmacy, College of Pharmacy, Hanyang University, Ansan 15588, Republic of Korea \\ ${ }^{2}$ Institute of Pharmacological Research, Hanyang University, Ansan 15588, Republic of Korea \\ ${ }^{3}$ South East Asia Regional Office, World Health Organization, New Delhi-110002, India
}

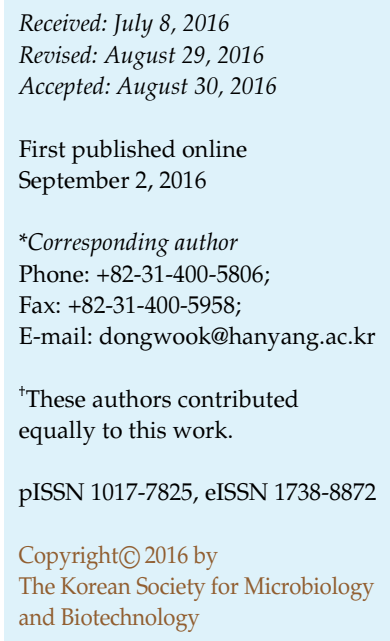

Vibrio cholerae O1 serogroup Wave $3 \mathrm{El}$ Tor strains are presently prevalent worldwide. The Wave 3 El Tor strains contain a TLC:RS1:CTX array on chromosome 1, and no element is integrated on chromosome 2. A multiplex PCR optimized to identify the TLC:RS1:CTX array of Wave 3 strains has been developed in this study. By using eight primers, the multiplex PCR can identify the characteristic CTX and RS1 array of Wave 3 strains from various arrays of strains belonging to other Waves. The four amplified DNA fragments of Wave 3 strains have been cloned in a vector, which could be used as a positive control for the multiplex PCR. This multiplex PCR and the positive control set could be useful tools for rapid recognition of Wave 3 El Tor strains.

Keywords: $V$. cholerae, multiplex PCR

\section{Introduction}

Vibrio cholerae serogroup $\mathrm{O} 1$ strains have been classified into two biotypes, classical and El Tor, based on their microbiological characteristics [10]. Strains belonging to each biotype contain biotype-specific cholera toxin phage (CTX), and thus produce biotype-specific cholera toxin (CT). CTX-cla contains $c t x B^{\text {cla }}(\operatorname{ctxB1})$, whereas CTX-1 contains $c t x B^{\mathrm{El} T o r}$ (ctxB3). The two cholera toxin types are distinguished by two amino acids difference (39th and 68th position) in the binding subunit (B subunit).

Classical biotype strains have been considered responsible for the first six cholera pandemics from the early 19th century $[5,10]$. Although various CTX arrays were suspected in classical strains, two most studied classical biotype strains (O395 and 569B) contained a TLC (toxin linked cryptic): ${ }^{\text {trunc }}$ CTX-cla:CTX-cla on chromosome 1 and a solitary CTX-cla on chromosome 2 [1].

El Tor biotype strains that have caused the current 7 th cholera pandemic are divided into several subgroups (Waves) by variations in the genome and the CTX phages they harbor [16]. Wave 1 strains, which had been prevalent worldwide from the beginning of the 7th cholera pandemic (early 1960s) to the 1990s, contain CTX-1 (CTX ${ }^{\mathrm{El}}$ Tor $)$ on chromosome 1, frequently together with a satellite phage, RS1. Generally, the Wave 1 strains do not contain any element on chromosome 2. Various CTX-1 and RS1 arrays, such as TLC:CTX-1:RS1, TLC:RS1:CTX-1:RS1, and TLC:CTX1:CTX-1:RS1, have been identified in the Wave 1 strains [7].

From the early 1990s, atypical El Tor strains that produce classical CT have emerged from the Ganges Delta area and have replaced the Wave 1 strains globally [11]. The Wave 1 strains have not been isolated, at least clinically, since the late 1990s. Atypical El Tor strains have been categorized into two main groups, Wave 2 and Wave 3 El Tor strains. Wave 2 strains typically contain a tandem repeat of CTX-2 on chromosome 2, and various arrays of CTX-1 and RS1 on chromosome 1 [13]. A genetic mechanism of the generation of Wave 2 strains from a Wave 1 strain, the acquisition of CTX-2 and gradual loss of CTX-1 and RS1, has been suggested [11, 12].

A TLC:RS1:CTX array has been identified in most Wave 3 
Table 1. V. cholerae strains used in this study.

\begin{tabular}{lllll}
\hline \multicolumn{1}{c}{ Strains } & \multicolumn{1}{c}{ Chromosome 1 } & Chromosome 2 & \multicolumn{1}{c}{ Biotype } & Genome sequence information \\
\hline N16961 & TLC:CTX-1:RS1 & None & Wave 1 El Tor & AE003852/AE003853 \\
O395 & TLC: ${ }^{\text {Trunc }}$ CTX-cla:CTX-cla & CTX-cla & Classical & CP000626/CP000627 \\
MG116025 & TLC:RS1:CTX-1:RS1 & None & Wave 2 El Tor & ERS013135 \\
V212-1 & TLC:RS1:CTX-1:RS1 & CTX-2:CTX-2 & Wave 2 El Tor & ERS013132 \\
B33 & No TLC, no element & CTX-2:CTX-2 & Wave 2 El Tor & ACHZ00000000 \\
IB4122 & TLC:RS1:CTX-3 & None & Wave 3 El Tor & ERS013264 \\
IB5230 & TLC:RS1CTX-3b & None & Wave 3 El Tor & AELH00000000.1 \\
\hline
\end{tabular}

strains [19]. The CTX phages in the Wave 3 strains are distinguished from CTX-1 in Wave 1 strains mainly by $\operatorname{ct} x B$; however, detailed sequence analysis showed that the CTX phages in the Wave 3 strains have been generated by homologous recombination between CTX-1 and RS1 [12]. CTX-3-CTX-6, depending on the recombination position, have been recognized among the Wave 3 strains. From 2006, variants of Wave 3 strains that contained a new type of $\operatorname{ct} x B, \operatorname{ct} x B 7$, have been spreading globally [3,11]. At least two new variant CTX phages (CTX-3b and CTX-6b) that contained $\operatorname{ct} x B 7$ have been noticed. Recent surveillance data showed that the strains containing $c t x B 7$ have replaced strains with $\operatorname{ctxB1}$ in India and other regions [11, 18, 24].

The global $V$. cholerae population seems to have changed regularly at 20- to 30-year intervals [11]. Wave 3 El Tor strains are prevailing worldwide, and a rapid and simple test to discriminate the Wave 3 strains from others is necessary to monitor the spread of Wave 3 strains and potential population changes of $V$. cholerae. A mismatchamplification mutation PCR assay (MAMA-PCR) and the double-mismatch-amplification mutation PCR assay (DMAMA-PCR) have been developed for discrimination of $\operatorname{ct} x B 1 / \operatorname{ct} x B 3$ and $\operatorname{ct} x B 1 / \operatorname{ct} x B 7$, respectively $[15,18]$. These methods have been proven to be valuable tools to trace the population changes $V$. cholerae [8]. However, a number of characteristics, such as variations in CTX phage genome, $\operatorname{tc} A$, and amount of cholera toxin production, have been shown to vary within Wave 3 strains $[8,17]$, which suggest that additional tests are required for the monitoring of current Wave 3 strains.

Various combinations of PCR have been developed for determination of the CTX and RS1 array in $V$. cholerae strains $[2,4,21]$. In this study, a multiplex PCR using four pairs of primers has been developed for rapid determination of the TLC:RS1:CTX array on chromosome 1 and no element on chromosome 2 in the Wave 3 strains. Various arrays of CTX and RS1 in Wave 1 and 2 El Tor strains and classical biotype strains could be discriminated by this multiplex PCR. The PCR products of each primer pair have been cloned into a vector, which could be used as a positive control.

\section{Materials and Methods}

\section{Bacterial Strains}

Seven $V$. cholerae strains were recruited for evaluation of the multiplex PCR developed in this study (Table 1). A Wave 1 El Tor strain, N16961, contained the TLC:CTX-1:RS1 array on chromosome 1. O395, a classical strain, contained the TLC, truncated CTX-cla, followed by an authentic CTX-cla on chromosome 1 and a solitary CTX-cla on chromosome 2. Three Wave 2 strains that contained different CTX array have been analyzed in this study. MG116025 contained a TLC:RS1:CTX-1:RS1 array on chromosome 1 and no element on chromosome 2. V212-1 and B33 contained a tandem repeat of CTX-2 on chromosome 2 in common; however, V212-1 contained the TLC:RS1:CTX-1:RS1 array on chromosome 1 and B33 contained no element. Wave $3 \mathrm{El}$ Tor strains that contained TLC:RS1:CTX-3 (IB4122) or TLC:RS1:CTX-3b (IB5230, a clinical isolate from the 2010 Haitian cholera outbreak) were included in this study.

\section{PCR Optimization}

Primers used in this study are listed in Table 2. A primer ctxAF2 was designed in this study and all other primers were previously reported for individual PCRs [4]. The PCR was performed in a $25 \mu \mathrm{l}$ reaction volume containing $2.5 \mu \mathrm{l}$ of $10 \times$ reaction buffer (20 mM Tris- $\mathrm{HCl}, \mathrm{pH} 8.0 ; 100 \mathrm{mM} \mathrm{KCl}, 0.1$ mM EDTA, 1 mM DTT, 0.5\% Tween 20, 0.5\% Nonidet P-40, 50\% glycerol), 1.25 U Takara Ex Taq DNA polymerase (TaKaRa Bio, Japan), $2 \mu \mathrm{l}$ of $2.5 \mathrm{mM}$ dNTPs, $2 \mu$ l of template (bacterial colony resuspended in $200 \mu \mathrm{l}$ of LB, or purified plasmid DNA), $8 \mu$ l of primers ( $1 \mu \mathrm{l}$ of each primer in various concentrations), and $10.25 \mu \mathrm{l}$ of distilled water. The PCR condition was predenaturation at $94^{\circ} \mathrm{C}$ for $5 \mathrm{~min}, 30$ cycles of denaturing at $95^{\circ} \mathrm{C}$ for $30 \mathrm{sec}$, annealing at $63^{\circ} \mathrm{C}$ for $30 \mathrm{sec}$, and elongation at $72^{\circ} \mathrm{C}$ for $3 \mathrm{~min}$, followed by an extension at $72^{\circ} \mathrm{C}$ for $10 \mathrm{~min}$. The annealing temperature was adjusted to $63^{\circ} \mathrm{C}$ from the best result of the reactions tested at $50-65^{\circ} \mathrm{C}$. The concentration of each primer was adjusted to 10,5 , and 2.5 pmol per microliter to optimize the reaction condition (Table 2). The MAMA-PCR, and 
Table 2. Primers used in this study.

\begin{tabular}{llcc}
\hline Primer & \multicolumn{1}{c}{ Primer sequence } & Amount used per reaction $(25 \mu \mathrm{l})$ & Reference \\
\hline Ch1F & GACCACTCAGGCCGCTGAAAT & $2.5 \mathrm{pmol}$ & {$[4]$} \\
Ch1R & CCGCGCTCAAGTGGTTATCGG & $5 \mathrm{pmol}$ & {$[4]$} \\
cepR1 & AAACAGCAAGAAAACCCCGAG & $10 \mathrm{pmol}$ & {$[4]$} \\
rstCF4 & AAATCCGCAACTCAAGGCATTGA & $5 \mathrm{pmol}$ & {$[4]$} \\
rstCR4 & TAAGCGCCTGAACGCAGATATAAAG & $5 \mathrm{pmol}$ & {$[4]$} \\
ctxAF2 & CTAGGTGTAAAATTCCTTGAC & $10 \mathrm{pmol}$ & This study \\
Ch2F & AACAACAGGTTGCAAGAGAGCATT & 5 pmol & {$[4]$} \\
Ch2R & TATTGCTTTTTAATGGCCGTT & 5 pmol & {$[4]$} \\
\hline
\end{tabular}

the DMAMA-PCR for discrimination of $\operatorname{ctxB1}, \operatorname{ct} x B 3$, and $\operatorname{ct} x B 7$ were performed as described previously $[15,18]$.

\section{Cloning of PCR Products}

The PCR-amplified product of the individual PCRs with the four primer sets (Ch1F/rstCR4, rstCF4/cepR, ctxAF2/Ch1R, and Ch2F/Ch2R) of a Wave 3 strain, IB4122, were purified using a Qiagen PCR purification spin kit (Qiagen, Germany), and cloned into a plasmid vector by using the Qiagen PCR cloning kit (Qiagen). These four recombinant plasmids were used as a positive control for the multiplex PCR of Wave $3 \mathrm{El}$ Tor strains. In addition to the four fragments amplified from Wave 3 strains, other DNA fragments amplified from various primer pairs in diverse arrays (Ch1F/cepR, rstCF4/Ch1R, ctxAF2/rstCR4, Ch2F/ cepR, ctxAF2/Ch2R, and ctxAF2/cepR) were also cloned into the pDrive vector.

\section{Results}

\section{Optimization of Multiplex PCR}

The possible amplified products from the multiplex PCR based on the primer annealing position and the CTX/RS1 element array of each strain are listed in Table 3. Four DNA fragments could be amplified by the combination of four primer pairs (Ch1F/rstCR4. rstCF4/cepR, ctxAF2/Ch1R, and Ch2F/Ch2R) from the TLC:RS1:CTX array on chromosome 1 and chromosome 2 of Wave 3 strains. Each individual PCR was shown to produce amplified fragments [19].

The multiplex PCR with the initial condition $(10 \mathrm{pmol}$ each primer and annealing temperature $58^{\circ} \mathrm{C}$ ) generated PCR products that do not coincide with the expected results when tested with one strain belonging to each Wave of El Tor strains (Fig. 1).

For example, five fragments (primer pairs Ch1F/cepR1, ctxAF2/rstCR4, ctxAF2/Ch1R, rstCF4/Ch1R, and Ch2F/ Ch2R) were expected from N16961. Although three amplified DNA bands (rstCF4/Ch1R of $630 \mathrm{bp}, \mathrm{Ch} 2 \mathrm{~F} /$ Ch2R of $910 \mathrm{bp}$, and Ch1F/cepR of 3,112 bp) were clearly
Table 3. Expected PCR products from the $V$. cholerae strains used in this study.

\begin{tabular}{|c|c|}
\hline \multirow[t]{5}{*}{ N16961 } & ctxAF2/Ch1R $(3,884$ bp $)$ \\
\hline & ctxAF2/rstCR4 (3,158bp) \\
\hline & Ch1F/cepR1 (3,112 bp) \\
\hline & Ch2F/Ch2R (910 bp) \\
\hline & rstCF4/Ch1R (630 bp) \\
\hline \multirow[t]{4}{*}{ IB4122 } & Ch1F/rstCR4 (3,078 bp) \\
\hline & rstCF4/cepR (2,663 bp) \\
\hline & ctxAF2/Ch1R (1,159 bp) \\
\hline & Ch2F/Ch2R (910 bp) \\
\hline \multirow[t]{4}{*}{ IB5230 } & Ch1F/rstCR4 (3,078 bp) \\
\hline & rstCF4/cepR (2,663 bp) \\
\hline & ctxAF2/Ch1R (1,159 bp) \\
\hline & Ch2F/Ch2R (910 bp) \\
\hline \multirow[t]{3}{*}{ B33 } & ctxAF2/cepR (3,324 bp) \\
\hline & Ch2F/cepR (2,904 bp) \\
\hline & $\operatorname{ctxAF} 2 / \mathrm{Ch} 2 \mathrm{R}(1,330 \mathrm{bp})$ \\
\hline \multirow[t]{3}{*}{ O395 } & Ch2F/cepR (2,904 bp) \\
\hline & ctxAF2/Ch2R (1,330 bp) \\
\hline & $\operatorname{ctxAF2/Ch1R~(1,159~bp)~}$ \\
\hline \multirow[t]{8}{*}{ V212-1 } & $\operatorname{ctxAF} 2 / \operatorname{Ch} 1 \mathrm{R}(3,884 \mathrm{bp})$ \\
\hline & ctxAF2/cepR (3,324 bp) \\
\hline & $\operatorname{ctxAF} 2 / \mathrm{rstCR} 4(3,158 \mathrm{bp})$ \\
\hline & Ch1F/rstCR4 (3,078 bp) \\
\hline & Ch2F/cepR $(2,904$ bp $)$ \\
\hline & $\mathrm{rstCF} 4 / \mathrm{cepR}(2,663 \mathrm{bp})$ \\
\hline & $\operatorname{ctxAF} 2 / \operatorname{Ch} 2 \mathrm{R}(1,330 \mathrm{bp})$ \\
\hline & rstCF4/Ch1R (630 bp) \\
\hline \multirow[t]{6}{*}{ MG116025 } & ctxAF2/Ch1R $(3,885 \mathrm{bp})$ \\
\hline & ctxAF2rstCR4 $(3,158 b p)$ \\
\hline & Ch1F/rstCR4 (3,078 bp) \\
\hline & rstCF4/cepR (2,663 bp) \\
\hline & Ch2F/Ch2R (910 bp) \\
\hline & $\mathrm{rstCF} 4 / \mathrm{Ch} 1 \mathrm{R}(630 \mathrm{bp})$ \\
\hline
\end{tabular}




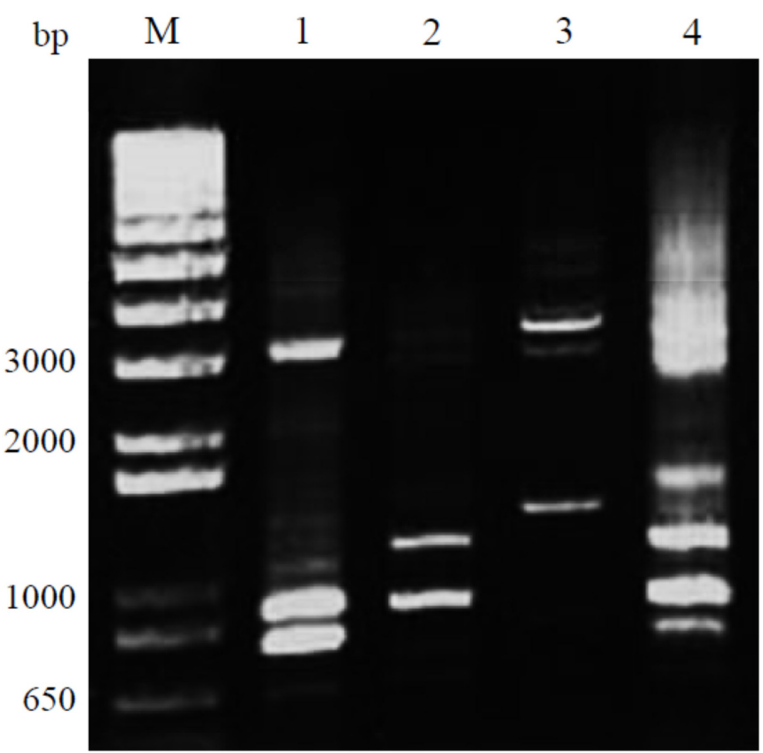

Fig. 1. Agarose gel electrophoresis of the multiplex PCR products.

The primer amount was adjusted at $10 \mathrm{pmol}$ per reaction. Strains N16961 (Wave 1, lane 1), IB4122 (Wave 3, lane 2), B33 (Wave 2, lane 3), and a mixture of three strains (lane 4 ) were used as the template. M: DNA size marker.

shown, the DNA fragments expected to be amplified by ctxAF2/rstCR4 and ctxAF2/Ch1R were not shown in the agrose gel (Fig. 1, lane 1). Similarly, four fragments $(3,078$, $2,663,1,159$, and $910 \mathrm{bp}$ ) were expected from the Wave 3 strain (IB4122), but two bands were observed on the agarose gel (Fig. 1, lane 2).

The PCR condition was optimized by varying the annealing temperature $\left(55-65^{\circ} \mathrm{C}\right)$ and the amount of each primer $(10,5$, and $2.5 \mathrm{pmol})$ with the bacterial resuspension as the template to generate the best results for Wave 3 strains. The final optimum reaction condition was determined to be the annealing temperature of $63^{\circ} \mathrm{C}$ and various amounts of each primer as shown in Table 2 (2.5 pmol for Ch1F, 10 pmol for cepR and ctxAF2, and 5 pmol for other primers per $25 \mu$ reaction), and four amplified DNA fragments were observed from Wave 3 strains (Fig. 2). Other strains showed differences in the agarose gel from the expected results; however, the multiple band pattern on the agarose gel was sufficiently diverse to discriminate the TLC:RS1:CTX array of Wave 3 strains from other strains.

\section{Construction of Positive Control Templates}

The DNA fragments from each individual PCR of Wave 3 strains were cloned in PCR cloning system. Four PCR amplified DNA fragments (amplified by primer pairs

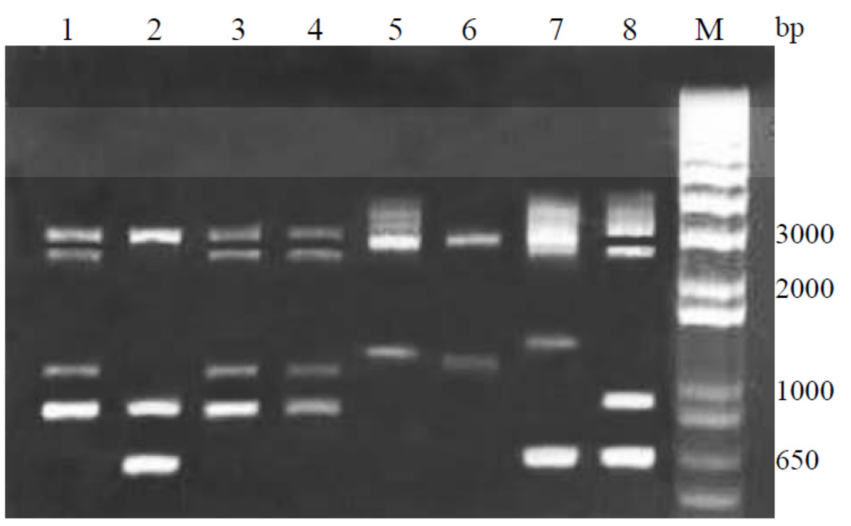

Fig. 2. Agarose gel electrophoresis of the multiplex PCR products in the optimized condition.

The PCR condition was optimized (described in the main text) for the PCR amplification of the Wave 3 strains. The mixture of four recombinant plasmids (pDrive vectors harboring each of DNA fragments amplified from Wave 3 strains) was used as a positive control (lane 1). Strains N16961 (Wave 1, lane 2), IB4122 (Wave 3, Lane 3), IB5230 (Wave 3, lane 4), B33 (Wave 2, lane 5), O395 (classical biotype strain, lane 6), V212-1 (Wave 2 strain, lane 7), and MG116025 (Wave 2, lane 8) were tested. M: DNA size marker.

Ch1F/rstCR4, rstCF4/cepR, ctxAF2/Ch1R, and Ch2F/ $\mathrm{Ch} 2 \mathrm{R})$ from the Wave 3 strain were cloned in the pDrive vector (Qiagen PCR cloning kit) to develop a positive control template for the multiplex PCR.

To set up the positive control, a mixture of $10 \mathrm{ng}$ of each plasmid (pDrive-Ch1F/rstR4, pDrive-rstCF4/cepR, pDrivectxAF2/Ch1R, and pDrive-Ch2F/Ch2R) was tested in the multiplex PCR; however, the efficiency of amplification of DNA fragment from each plasmid varied. Therefore, the amount of each plasmid was adjusted to give the best results in the multiplex PCR condition that was optimized for the bacterial resuspension. The optimum amount of each plasmid was determined as $5 \mathrm{ng}$ for pDrive-Ch1F/ rstCR4 and pDrive-rstCF4/cepR, and $10 \mathrm{ng}$ for pDrivectxAF2/Ch1R and pDrive-Ch2F/Ch2R, for $25 \mu \mathrm{l}$ multiplex PCRs (Fig. 2, lane 1).

\section{MAMA-PCR and DMAMA PCR}

MAMA-PCR to discriminate ctxB1 (classical $c t x B$ ) and ct $x B 3$ (El Tor $c t x B$ ) and DMAMA-PCR to discriminate $c t x B 7$ and $c t x B 1$ were applied to the $V$. cholerae strains $[15,18]$. As shown in Fig. 3, two PCR sets specifically amplified typespecific $\operatorname{ct} x B$. The $\operatorname{ct} x B 3$-specific fragment was amplified in MAMA-PCR from the strains N16961 and MG116025, the ct $x$ B1-specific fragment was amplified from strains O395, B33, and IB4122, and ctxB7 was amplified from strain 
A

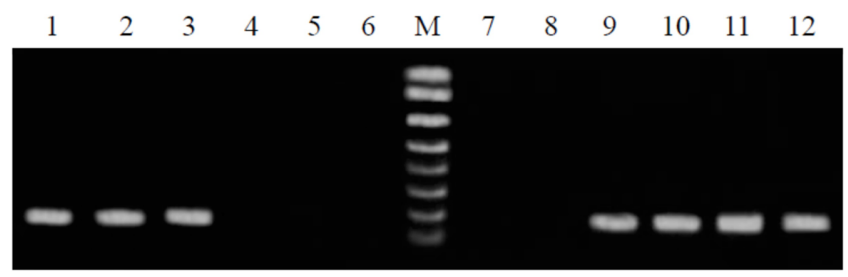

B

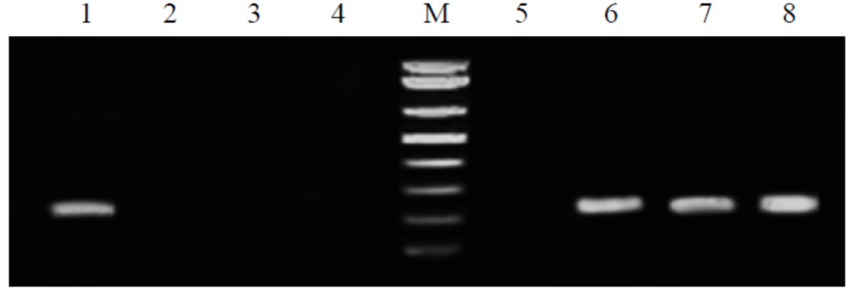

Fig. 3. MAMA (A) and DMAMA (B) PCR assays of six reference strains used in this study.

(A) MAMA-PCR with a primer combination FW con/RV El for detection of $c t x B 3$ (lanes 1-6) and a primer combination FW con/RV $\mathrm{Cla}$ for detection of $c t x B 1$ (lanes 7-12). Amplified DNA fragment of $186 \mathrm{bp}$ is shown in each strain. The strain V212-1 contained both $c t x B 3$ (El Tor type $c t x B$, lane 3) and $c t x B 1$ (classical type $c t x B$, lane 9). Lanes 1 and 7, strain N16961; lanes 2 and 8, strain MG116025; lanes 3 and 9, strain V212-1; lanes 4 and 10, strain O395; lanes 5 and 11, strain B33; lanes 6 and 12, strain IB4122. M: DNA size marker (1000, 850, 650, 500, $400,300,200$, and $100 \mathrm{bp}$ standard size marker fragments are shown). (B) DMAMA-PCR with the primer combination $\mathrm{ct} \times \mathrm{BF} 3 / \mathrm{Cla}$ for detection of $c t x B 7$ (lanes 1-4) and the primer combination ctxBF4/Cla for detection of ctxB1 (lanes 5-8). A $191 \mathrm{bp}$ DNA fragment was amplified from the DMAMA-PCR. Lanes 1 and 5, IB5230; lanes 2 and 6, O395; lanes 3 and 7, B33; and lanes 4 and 8; IB4122.

IB5230. As expected, both $c t x B 1$ - and $c t x B 3$-specific fragments were amplified from strain V212-1, which contained CTX-1 and CTX-2.

\section{Discussion}

The global population of $V$. cholerae O1 serogroup El Tor strains has changed at least twice since the first emergence of prototype Wave 1 El Tor strains in the early 1960s [11, 23]. The Wave 1 strains has been replaced by Wave 2 and 3 strains in the 1990s, which could be characterized by the change of CTX phage and the $c t x B$ they contained. The Wave 2 strains have diminished, while the Wave 3 strains have remained prevalent [16]. The Wave 2 and 3 strains have been called atypical El Tor strains, since they produced the classical biotype cholera toxin. It is perhaps not appropriate to describe the atypical El Tor strains as atypical, since they have entirely replaced the prototype El Tor strain. Therefore, a new description as Wave has been used to distinguish the atypical strains from the prototype El Tor strains [16, 22].

Diverse CTX/RS1 arrays in El Tor biotype strains have been described; however, current Wave 3 El Tor strains contain a simple uniform array on chromosome 1, TLC:RS1:CTX. A multiplex PCR to determine this array has been developed in this study by utilizing eight primers, which showed distinct amplified DNA patterns compared with various CTX/RS1 arrays of strains belonging to other Waves.

The eight primers designed in this study can amplify virtually all the possible CTX/RS1 arrays found in V. cholerae strains. Ch1F, Ch2F, cepR, and rstCR4 can identify if the array on chromosomes 1 and 2 starts with either CTX or RS1. Similarly, ctxAF2, rstCF4, Ch1R, and Ch2R can determine if the array ends with CTX or RS1 on chromosomes 1 and 2. The CTX:RS1, RS1:CTX, CTX:CTX, and RS1:RS1 arrays can be determined by the primer pairs ctxAF2/rstCR4, rstCF4/cepR, ctxAF2/cepR, and rstCF4/ rstCR4, respectively. Ch1F/Ch1R and $\mathrm{Ch} 2 \mathrm{~F} / \mathrm{Ch} 2 \mathrm{R}$ can determine that no element is incorporated in the CTX integration loci on chromosomes 1 and 2. The multiplex PCR condition described in this report is optimized for the recognition of TLC:RS1:CTX on chromosome 1 and no element on chromosome 2 . The reaction condition could be further modified for better results for other arrays.

A number of detailed features of the CTX/RS1 array cannot be determined by these primers. Although various arrays can be determined by the combinations of primers listed in this study, the multiplex PCR cannot provide information if a particular array is repeated or present in multiple positions. Some strains, including B33 in this study, have lost the TLC element and Ch1F cannot be used in these strains. Alternatively, primer Ch1F0 (GCG AGT AAT GTT GAG CAT TTT CCT CAC) could be used to determine the CTX array in strains that have lost the TLC element [4].

Although the Wave 3 strains have been prevalent worldwide during the last decade, the global $V$. cholerae population may change sooner or later [11]. Multiple genotyping methods have been developed to discriminate the $V$. cholerae strains, such as MLVA, $c t x B$ typing, and genome sequencing $[6,9,14,20]$. To monitor the population change, whole-genome sequencing may be the ultimate choice; however, a simple method is also necessary for rapid identification. The multiplex PCR developed in this 
study to identify the TLC:RS1:CTX array, together with DMAMA-PCR, which can distinguish the $c t x B$ type, could be applied for rapid recognition of Wave $3 \mathrm{El}$ Tor strains in a laboratory where a regular PCR is routinely performed.

A few notable genetic characters within Wave 3 strains have been described; namely, the presence of variation in VSP-2 (Vibrio seventh pandemic island) and SXT, and some SNPs in multiple genes such as $r t x A$ and $\operatorname{tcp} A[3,16,25,26]$.

A further genotyping method for detailed characterization of clinical isolates could be developed by targeting these specific traits for additional confirmation of the identification of Wave 3 strains.

\section{Acknowledgments}

This work was supported by the research fund of Hanyang University (HY-2012-P).

\section{References}

1. Basu A, Mukhopadhyay AK, Garg P, Chakraborty S, Ramamurthy T, Yamasaki S, et al. 2000. Diversity in the arrangement of the CTX prophages in classical strains of Vibrio cholerae O1. FEMS Microbiol. Lett. 182: 35-40.

2. Bhuiyan NA, Nusrin S, Ansaruzzaman M, Islam A, Sultana M, Alam M, et al. 2012. Genetic characterization of Vibrio cholerae O1 strains isolated in Zambia during 1996-2004 possessing the unique VSP-II region of El Tor variant. Epidemiol. Infect. 140: 510-518.

3. Chin CS, Sorenson J, Harris JB, Robins WP, Charles RC, Jean-Charles RR, et al. 2011. The origin of the Haitian cholera outbreak strain. N. Engl. J. Med. 364: 33-42.

4. Choi SY, Lee JH, Kim EJ, Lee HR, Jeon YS, von Seidlein L, et al. 2010. Classical RS1 and environmental RS1 elements in Vibrio cholerae $\mathrm{O} 1 \mathrm{El}$ Tor strains harbouring a tandem repeat of CTX prophage: revisiting Mozambique in 2005. J. Med. Microbiol. 59: 302-308.

5. Devault AM, Golding GB, Waglechner N, Enk JM, Kuch M, Tien $\mathrm{JH}$, et al. 2014. Second-pandemic strain of Vibrio cholerae from the Philadelphia cholera outbreak of 1849. N. Engl. J. Med. 370: 334-340.

6. Dolores J, Satchell KJ. 2013. Analysis of Vibrio cholerae genome sequences reveals unique $r t x A$ variants in environmental strains and an $r t x A$-null mutation in recent altered El Tor isolates. mBio 4: e00624.

7. Faruque SM, Asadulghani, Kamruzzaman M, Nandi RK, Ghosh AN, Nair GB, et al. 2002. RS1 element of Vibrio cholerae can propagate horizontally as a filamentous phage exploiting the morphogenesis genes of СТХФ. Infect. Immun. 70: 163-170.

8. Ghosh P, Naha A, Basak S, Ghosh S, Ramamurthy T, Koley
$\mathrm{H}$, et al. 2014. Haitian variant tcpA in Vibrio cholerae O1 El Tor strains in Kolkata, India. J. Clin. Microbiol. 52: 1020-1021.

9. Ghosh R, Nair GB, Tang L, Morris JG, Sharma NC, Ballal M, et al. 2008. Epidemiological study of Vibrio cholerae using variable number of tandem repeats. FEMS Microbiol. Lett. 288: 196-201.

10. Kaper JB, Morris JG Jr, Levine MM. 1995. Cholera. Clin. Microbiol. Rev. 8: 48-86.

11. Kim EJ, Lee CH, Nair GB, Kim DW. 2015. Whole-genome sequence comparisons reveal the evolution of Vibrio cholerae O1. Trends Microbiol. 23: 479-489.

12. Kim EJ, Lee D, Moon SH, Lee CH, Kim SJ, Lee JH, et al. 2014. Molecular insights into the evolutionary pathway of Vibrio cholerae O1 atypical El Tor variants. PLoS Pathog. 10: e1004384.

13. Lee JH, Choi SY, Jeon YS, Lee HR, Kim EJ, Nguyen BM, et al. 2009. Classification of hybrid and altered Vibrio cholerae strains by CTX prophage and RS1 element structure. J. Microbiol. 47: 783-788.

14. Li B, Chen R, Wang D, Tan H, Ke B, He D, et al. 2016. Distribution and molecular characteristics of Vibrio cholerae O1 El Tor isolates recovered in Guangdong Province, China, 1961-2013. Infect. Genet. Evol. 37: 70-76.

15. Morita M, Ohnishi M, Arakawa E, Bhuiyan NA, Nusrin S, Alam M, et al. 2008. Development and validation of a mismatch amplification mutation PCR assay to monitor the dissemination of an emerging variant of Vibrio cholerae $\mathrm{O} 1$ biotype El Tor. Microbiol. Immunol. 52: 314-317.

16. Mutreja A, Kim DW, Thomson NR, Connor TR, Lee JH, Kariuki S, et al. 2011. Evidence for several waves of global transmission in the seventh cholera pandemic. Nature 477: 462-465.

17. Naha A, Chowdhury G, Ghosh-Banerjee J, Senoh M, Takahashi T, Ley B, et al. 2013. Molecular characterization of high-level-cholera-toxin-producing El Tor variant Vibrio cholerae strains in the Zanzibar Archipelago of Tanzania. J. Clin. Microbiol. 51: 1040-1045.

18. Naha A, Pazhani GP, Ganguly M, Ghosh S, Ramamurthy T, Nandy RK, et al. 2012. Development and evaluation of a PCR assay for tracking the emergence and dissemination of Haitian variant $\operatorname{ct} x B$ in Vibrio cholerae $\mathrm{O} 1$ strains isolated from Kolkata, India. J. Clin. Microbiol. 50: 1733-1736.

19. Nguyen BM, Lee JH, Cuong NT, Choi SY, Hien NT, Anh DD, et al. 2009. Cholera outbreaks caused by an altered Vibrio cholerae $\mathrm{O} 1 \mathrm{El}$ Tor biotype strain producing classical cholera toxin B in Vietnam in 2007 to 2008. J. Clin. Microbiol. 47: 1568-1571.

20. Okada K, Roobthaisong A, Nakagawa I, Hamada S, Chantaroj S. 2012. Genotypic and PFGE/MLVA analyses of Vibrio cholerae O1: geographical spread and temporal changes during the 2007-2010 cholera outbreaks in Thailand. PLoS One 7: e30863.

21. Raychoudhuri A, Mukherjee P, Ramamurthy T, Nandy RK, 
Takeda Y, Nair GB, Mukhopadhyay AK. 2010. Genetic analysis of CTX prophages with special reference to $\operatorname{ct} x B$ and $r s t R$ alleles of Vibrio cholerae $\mathrm{O} 139$ strains isolated from Kolkata over a decade. FEMS Microbiol. Lett. 303: 107-115.

22. Raychoudhuri A, Mukhopadhyay AK, Ramamurthy T, Nandy RK, Takeda Y, Nair GB. 2008. Biotyping of Vibrio cholerae O1: time to redefine the scheme. Indian J. Med. Res. 128: 695-698.

23. Safa A, Nair GB, Kong RY. 2010. Evolution of new variants of Vibrio cholerae O1. Trends Microbiol. 18: 46-54.

24. Shakya G, Kim DW, Clemens JD, Malla S, Upadhyaya BP, Dumre SP, et al. 2012. Phenotypic and genetic characterization of Vibrio cholerae O1 clinical isolates collected through national antimicrobial resistance surveillance network in Nepal. World J. Microbiol. Biotechnol. 28: 2671-2678.

25. Spagnoletti M, Ceccarelli D, Colombo MM. 2012. Rapid detection by multiplex PCR of genomic islands, prophages and integrative conjugative elements in $V$. cholerae 7 th pandemic variants. J. Microbiol. Methods 88: 98-102.

26. Zhou H, Cui Z, Diao B, Zhang C, Pang B, Zhang L, Kan B. 2013. A three-loci variable number of tandem repeats analysis for molecular subtyping of Vibrio cholerae $\mathrm{O} 1$ and O139. Foodborne Pathog. Dis. 10: 723-730. 